\title{
Attenuation of Activated elF2a Signaling by ISRIB Treatment After Spinal Cord Injury Improves Locomotor Function
}

\author{
Lei Chang ${ }^{1} \cdot$ Xiangyang Liu $^{1}$ - Jing Chen ${ }^{2} \cdot$ Hongzhe Liu $^{1} \cdot$ Guoping Wang ${ }^{1} \cdot$ Guohua Wang $^{1} \cdot$ Xiaoyun Liao $^{3}$. \\ Xiongjie Shen ${ }^{1} \mathbb{D}$
}

Received: 5 May 2021 / Accepted: 22 September 2021 / Published online: 13 October 2021

(C) The Author(s) 2021

\begin{abstract}
Following spinal cord injury (SCI), multiple signaling cascades are activated instantaneously in the injured segments of the spinal cord to create a complex and pathogenic microenvironment, making it difficult to treat SCI. Nevertheless, the significance of the integrated stress response (ISR) to the series of physiological and pathological changes that occur after SCI remains unclear. Through western blotting (WB), we determined that the autophosphorylation of stress receptors (GCN2, PERK, PKR, and HRI) was enhanced after SCI, leading to increased phosphorylation of eIF2 $\alpha$ at Ser51. Strikingly, we found that eIF2 $\alpha$ was highly phosphorylated at 1 day post injury (dpi) and that this hypophosphorylation was maintained thereafter in the spinal cord, especially in neurons, which suggests that intervening with eIF2 $\alpha$ phosphorylation may be a treatment strategy for SCI. Therefore, we employed the small molecule ISRIB, which inhibits eIF2 $\alpha$ phosphorylation when the ISR is activated at moderate or low levels but not when the ISR is highly activated. Daily intraperitoneal injection of ISRIB significantly inhibited ISR signaling after SCI, reduced the cytosolic localization of RNA-binding proteins, and decreased neuronal apoptosis. Histological and functional experiments further demonstrated that treatment with ISRIB after SCI effectively curbed morphological deterioration and promoted the recovery of locomotor function. In summary, the ISR plays an important role in SCI, and ISRIB is a promising drug for the treatment of SCI.
\end{abstract}

Keywords Integrated stress response $\cdot \operatorname{eIF} 2 \alpha \cdot$ Apoptosis $\cdot$ Locomotor function

$\begin{array}{ll}\text { Abbreviations } \\ \text { SCI } & \text { Spinal cord injury } \\ \text { CNS } & \text { Central nervous system } \\ \text { ISR } & \text { Integrated stress response } \\ \text { TC } & \text { Ternary complex } \\ \text { UPR } & \text { Unfolded protein reaction } \\ \text { ERSR } & \text { Endoplasmic reticulum stress response } \\ \text { SGs } & \text { Stress granules }\end{array}$

Xiongjie Shen

xjsrhpph@protonmail.com

1 Department of Spine Surgery, Hunan Provincial People's Hospital, The First Affiliated Hospital of Hunan Normal University), No.61, West Jiefang Road, Changsha 410005, China

2 Department of Endocrinology, Hunan Provincial People's Hospital, The First Affiliated Hospital of Hunan Normal University), Changsha, China

3 Department of Anesthesiology, Hunan Provincial People's Hospital, The First Affiliated Hospital of Hunan Normal University), Changsha, China

$\begin{array}{ll}\text { H\&E } & \text { Hematoxylin \& eosin } \\ \text { dpi } & \text { Days post injury } \\ \text { C-Cas3 } & \text { Cleaved-caspase 3 } \\ \text { C-Cas12 } & \text { Cleaved-caspase 12 } \\ \text { Cas12 } & \text { Caspase 12 }\end{array}$

\section{Introduction}

As one of the most serious public health problems worldwide, spinal cord injury (SCI) has serious physical and psychological impacts and imposes a heavy burden on society (Rubiano et al. 2015; Wagner et al. 2018). Since neurons in the mammalian central nervous system (CNS) cannot regenerate, the treatment of CNS injury is a challenge (Griffin 2003; Miller et al. 2016). In addition to irreversible necrosis caused by transient injury, secondary pathophysiological changes resulting from injury, such as inflammation (Tsarouchas et al. 2018), oxidative damage (Wang et al. 2019), and proteostasis imbalance (Valenzuela et al. 2012), can worsen the injury and increase mortality by causing loss of neurons and dysfunction 
of neural network connections. Therefore, a large number of studies have focused on secondary injury after SCI to identify potential therapeutic targets to curb the progression of the disease.

The integrated stress response (ISR) is a conservative defense response (Kroemer et al. 2010; Costa-Mattioli and Walter 2020; Kong et al. 2020) to proteostasis defects, nutritional deprivation, viral infection, redox imbalance, etc. The initiation of the ISR depends on the phosphorylation of Ser51 in eIF2 $\alpha$, which is induced by PERK, PKR, GCN2, and HRI (Malvezzi et al. 2020). Normally, the eIF2 ternary complex (TC), which consists of eIF2 (including the $\alpha, \beta$, and $\gamma$ subunits), GTP, and Met-tRNAi, participates in elongation during the translation of genes starting with AUG (Costa-Mattioli and Walter 2020). Phosphorylated eIF2 $\alpha$ can serve as a noncompetitive inhibitor of eIF2B to inhibit eIF2B-catalyzed dissociation of GDP from inactivated eIF2, thus blocking global protein translation. The transient inhibition of protein translation is conducive to mobilizing the cell's armory against stress, while chronic inhibition contributes to decreasing neurogenesis and impairing neuronal activity (Surget et al. 2011). In addition, the phosphorylation of eIF $2 \alpha$ can trigger the nuclear translocation of ATF4 and then promote the expression of stress resistance genes or proapoptotic genes (Zhou et al. 2020).

Appropriate phosphorylation of eIF $2 \alpha$ contributes to preserving cellular homeostasis in the context of cellular stress by limiting protein synthesis and promoting the translation of specific mRNAs to produce proteins involved in autophagy, the unfolded protein response (UPR), and heat shock (Zhu et al. 2019). Additionally, the expression of the apoptotic gene CHOP can be upregulated to induce apoptosis of damaged cells when activation of the ISR fails to resolve stress (Liu and Ye 2011; Arai et al. 2020). Nevertheless, existing evidence has demonstrated that the proteostasis imbalance-induced endoplasmic reticulum stress response (ERSR) can significantly affect the fate of neurons after SCI (Wang et al. 2017; Li et al. 2019), but the role and underlying mechanism of the ISR after SCI remain unclear.

Considering the dual role of the ISR in stress relief and apoptosis promotion, the significance of the ISR after SCI should be urgently explored. Here, we employed the small molecular compound ISRIB, which can cross the bloodbrain barrier and promote the assembly of eIF2B to catalyze the dissociation of GDP from the inactivated TC to inhibit the ISR signaling pathway (Zyryanova et al. 2018; Rabouw et al. 2019). Since ISRIB works by promoting eIF2B assembly, it fails to inhibit ISR when unassembled eIF2B is consumed by highly phosphorylated eIF $2 \alpha$. Thus, the ISR is not inhibited by ISRIB when it is overactivated, while the ISR is blocked by ISRIB when it is activated at a moderate or low level. Therefore, whether the application of ISRIB is beneficial for the survival of neurons after secondary injury and the improvement of behavioral function after SCI remains to be studied.

\section{Materials and Methods}

\section{Animal and Ethic Statement}

A total of 78 healthy male C57BL/6JNifdc mice (8-weekold, 20--23 g) were used in our study. The mice are housed in a clean grade environment with $12 \mathrm{~h}$ light and dark cycle $\left(23{ }^{\circ} \mathrm{C}, 60 \%\right.$ humidity), and they have free access to water and food. All experimental procedures were approved by Medical Ethics Committee of Hunan People's Hospital (First Affiliated Hospital of Hunan Normal University, 2019-41) and were performed in accordance with the National Institutes of Health guide for the care and use of Laboratory animals.

\section{Regents and Antibodies}

Anti-PKR (Cat. No. 18244-1-AP), anti-ATF4 (Cat. No. 10835-1-AP), anti-histone H3 (Cat. No. 17168-1-AP), antiTIA-1 (Cat. No. 12133-2-AP), anti-FUS (Cat. No. 115701-AP), anti-phospho-eIF2S1 (Ser51) (Cat. No. 28740-1AP), anti-eIF2 $\alpha$ (Cat. No. 11170-1-AP), anti-GAPDH (Cat. No. 60004-1-Ig), anti-GADD34 (Cat. No. 10449-1-AP), and anti-CHOP (Cat. No. 15204-1-AP) antibodies were purchased from Proteintech (Chicago, USA). Anti-GCN2 (Cat. No. PA5-105886) antibody was purchased from Cell Signaling Technology (Danvers, MA, USA). Anti-caspase 12 antibody (Cas12, Cat. No. 3282-100) was obtained from BioVision (San Francisco, USA). Anti-PERK (Cat. No. SC377400), anti-HRI (Cat. No. SC-365239), and anti-TDP-43 (Cat. No. SC-376311) antibodies were purchased from Santa Cruz Biotechnology (Dallas, TX, USA). Anti-phospho-PERK (Thr980) (Cat. No. BS-3330R) antibody was purchased from Bioss (Beijing, China). Antipuromycin (Cat. No. MABE343) antibody and puromycin (Cat. No. 540222) were obtained from Merck (Darmstadt, Germany). Anti-phospho-GCN2 (Thr899) (Cat. No. PA5105886) and anti-phosphor-PKR (Thr446 + Thr451) (Cat. No. BS-3337R) antibodies were obtained from Thermo Fisher Scientific (Waltham, MA, USA). Anti-NeuN (Cat. No. ab104224) and anti-cleaved caspase-3 (C-Cas3, Cat. No. ab49822) antibodies, Alexa Fluor 488-labeled or Alexa Fluor 647-labeled goat anti-Rabbit or mouse secondary antibodies, and Bradford (Cat. No. ab119216) were purchased from Abcam (Cambridgeshire, England). Goat anti-rabbit/ mouse $\operatorname{IgG}(\mathrm{H}+\mathrm{L}) \mathrm{HRP}$ were obtained from MultiSciences (Hangzhou, Zhejiang, China). DAPI-containing fluorescent mounting media (Cat. No. 36308ES20) were purchased from YEASEN (Shanghai, China). Hematoxylin and eosin (H\&E) 
staining kit (Cat. No. G1120) was obtained from Solarbio (Beijing, China). Loading buffer (Cat. No. P0015L), antigen repair solution (Cat. No. P0083), and protein inhibitors (Cat. No. P1005) were obtained from Beyotime (Shanghai, China). Lysis buffer (Cat. No. AR0101) was purchased from BOSTER (Wuhan, Hubei, China). Bovine serum albumin (Catalog No. A7030) was purchased from Sigma (Shanghai, China).

\section{SCI Model and Drug Treatment}

Mice receiving SCI and sham surgery were anesthetized with $4 \%$ isoflurane and then maintained with $1.5 \%$ isoflurane. For the SCI group, after the laminectomy, the exposed T9 segment of spinal cord suffered moderate contusion (10 $\mathrm{g}, 1.5 \mathrm{~cm}$ ) as previously described (Liu et al. 2015, 2018), while the sham group only received laminectomy. Heating pads were applied to maintain body temperature $\left(37^{\circ} \mathrm{C}\right)$ during and after surgery. After SCI, bladders were manually expressed twice daily until a reflex bladder was established (12-16 days after SCI). ISRIB (Cat. No. HY-12495, MedChemExpress, Monmouth Junction, USA) was prepared and injected intraperitoneally $(2.5 \mathrm{mg} / \mathrm{kg} /$ day $)$ into mice after SCI as previously described (Krukowski et al. 2020).

\section{BMS Scoring System and Footprint Analysis}

In order to assess the recovery of locomotion function of injured mice, BMS scoring was conducted at $0,1,3,7,14$, and 28 days post injury (dpi) as previously described (Wang et al. 2018). Briefly, the mice were placed in an open field with a diameter of 42 inches and observed for 4 min by a well-trained observer who was blind to the experimental group. The scoring range is $0-9(0$, complete hind limb paralysis; 9, normal exercise). This is based on hind limb movement in an open field, including hind limb joint movement, weight support, plantar stepping, coordination, paw position, trunk, and tail control. After the BMS scoring, the mice hind limbs were immersed in red ink, and then the movement track of the mice was collected on the white runway for footprint analysis. Six independent mice were used for both foot imprinting and BMS scoring, and each mouse was tested 3 times to ensure accuracy.

\section{Tissue Preparation and Histopathologic Analysis}

At the indicated time point $(1,3,7$, and $28 \mathrm{dpi})$, the mice were sacrificed by carbon dioxide inhalation. For western blotting (WB) analysis, after the sacrificed mice were perfused with saline in the left ventricle, a total of $1 \mathrm{~cm}$ of spinal cord tissue above and below the epicenter was obtained. For immunofluorescence (IF) staining and H\&E staining, after the sacrificed mice were perfused with paraformaldehyde
(4\%) in the left ventricle, a total of $1 \mathrm{~cm}$ of spinal cord tissue above and below the epicenter was immersed in paraformaldehyde (4\%) for $48 \mathrm{~h}$ and then dehydrated and embedded in paraffin. Transverse sections of spinal cord $(5 \mu \mathrm{m})$ were obtained and loaded on glass slides. H\&E staining was performed according to the guidance of its manual after footprint analysis (Cat. No. G1120, Solarbio). The tissue section samples of each group in H\&E were derived from 6 independent mice.

\section{Extraction of Nuclear Proteins and Cytoplasmic Proteins}

To determine the distribution of transcription factor ATF4 and stress granules (SGs) associated proteins (TIA-1, FUS, and TDP-43) in the nucleus and cytoplasm, the nuclear proteins and cytoplasmic proteins in spinal cord tissue obtained above at 7 dpi were extracted by a nuclear protein and cytoplasmic protein extraction kit (Cat. No. P0027, Beyotime). The purity of the extracted nuclear components and cytoplasmic components were determined by detecting the expression levels of histone $\mathrm{H} 3$ (nuclear protein) and GAPDH (cytoplasmic protein).

\section{Measurement of Protein Synthesis}

In order to define the variations of protein synthesis around epicenter of spinal cord after SCI, puromycin was applied to label nascent peptides. Before being sacrificed, the mice were injected intraperitoneally with $0.04 \mathrm{mM} / \mathrm{kg}$ puromycin for $30 \mathrm{~min}$ as previously described (Park et al. 2014). Next, the expression level of puromycin-labeled proteins around epicenter of spinal cord was detected through WB analysis with anti-puromycin antibody.

\section{WB Analysis}

The spinal cord tissue obtained above for total proteins and puromycin-labeled proteins measurement were homogenized and lysed at $4{ }^{\circ} \mathrm{C}$ in the presence of lysis buffer and protease inhibitors. Bradford was subsequently used to measure the concentration of protein in tissue lysates. A total of $40 \mu \mathrm{g}$ of protein was separated by electrophoresis on sodium dodecyl sulfate-polyacrylamide gel in the presence of loading buffer and then transferred to polyvinylidene fluoride membrane (Cat. No. 1620256, Bio-Rad, Berkeley, CA, USA). Next, the membranes were blocked with skimmed milk (5\%) for $1 \mathrm{~h}$ at room temperature, followed by incubated with specific primary antibodies overnight at $4{ }^{\circ} \mathrm{C}$. Subsequently, the membranes were incubated with horseradish peroxidaseconjugated secondary antibodies at room temperature for 1 h. ChemiDicTM XRS+ Imaging System (Bio-Rad, Berkeley, CA, USA) was applied to visualized signals, and the 
signals were quantified by the ImageJ software. The protein samples of each group in WB came from 3 independent mice, and the samples of each mouse were tested three times to obtain the average value.

\section{IF Staining}

The sections (1,3, and $7 \mathrm{dpi})$ obtained above were dewaxed and gradient hydrated and then subjected to high-pressure antigen repair in the presence of antigen retrieval solution. The sections were then blocked with bovine serum albumin (5\%) at room temperature for $1 \mathrm{~h}$, followed by incubating overnight at $4{ }^{\circ} \mathrm{C}$ with specific antibodies. Next, the sections were incubated with Alexa Fluor 488-labeled or Alexa Fluor 647-labeled goat anti-rabbit or mouse antibodies for 1 $\mathrm{h}$ at $37^{\circ} \mathrm{C}$ and mounted with DAPI-containing fluorescent mounting media. In order to eliminate the misleading caused by non-specific staining, we added a negative control group (only incubate the secondary antibody of the corresponding species without incubating the primary antibody) in each experiment to ensure the specificity of the antibodies. Zeiss LSM710 microscope was used to capture images. In order to analyze the changes in fluorescence intensity, we first determined the range of neuron cell bodies through the NeuN channel and then employed ImageJ to determine the area of this range and measure the total fluorescence intensity of p-eIF $2 \alpha$ or C-Cas 3 in this area. The average fluorescence intensity is determined by calculating the ratio of the sum of the fluorescence intensity to the area, which is then used for statistics.

\section{Statistical Analysis}

All data were expressed as the mean \pm SEM. The normality of collected data was first determined by Shapiro-Wilk test. Then, the significant difference among multiple groups was measured by one-way analysis of variance (ANOVA) test, followed by Tukey's multiple comparison test to exhibit the difference between each two groups. The significant difference between SCI group and SCI+ISRIB group in BMS scoring was measured by Mann-Whitney test. The statistical evaluation of our data was performed in GraphPad Prism 8 Software. $P<0.05$ was considered statistically different. Except for BMS scoring and footprint, other experiments were repeated three times independently to ensure accuracy.

\section{Results}

\section{Activation of the ISR in Spinal Cord After SCI}

To define the significance of the ISR after SCI, the activation of four arms of the ISR, i.e., PERK, GCN2, HRI, and
PKR, was assessed by determining the phosphorylation state of these proteins at 1,3 , and 7 days post injury (dpi). Western blotting (WB) revealed that phosphorylation of PERK at Thr980 and the phosphorylation of PKR at Thr446 and Thr451 were significantly increased after SCI and peaked at 3 dpi (Fig. 1A, B, and E). Additionally, the phosphorylation of HRI and the phosphorylation GCN2 at Thr899 were also enhanced after SCI, but they peaked at $1 \mathrm{dpi}$ (Fig. 1A, C, and $\mathrm{D}$ ). These data suggest that the ISR is activated in the injured segments of the spinal cord.

\section{Persistent Activation of the ISR in Neurons After SCI}

Next, the initiation of downstream signaling of these four arms was detected by WB and IF staining. The phosphorylation of eIF2 $\alpha$ at Ser51 is the core event of ISR activation. Here, the results revealed a significant increase in the phosphorylation level of eIF $2 \alpha$ observed at $1 \mathrm{dpi}$, and there was still a significant difference at 7 dpi (Fig. 2A, B). Especially in NeuN-positive cells (neuron), the phosphorylation level of eIF2 $\alpha$ was still significantly higher in the SCI group than in the sham group at 7 dpi (Fig. 2C, D), revealing that the ISR was activated around the epicenter of the spinal cord and that ISR activation was not temporary after SCI.

\section{ISRIB Treatment Reverses SCI-Induced Apoptosis}

The ISR inhibitor ISRIB was then employed to better determine the significance of the ISR after SCI. WB revealed that daily administration of ISRIB decreased the expression level of the eIF $2 \alpha$ downstream protein GADD34 and the apoptotic protein $\mathrm{CHOP}$ and attenuated the cleavage of the proteotoxicity-associated apoptotic protein caspase 12 (Cas12) in the spinal cord at 7 dpi (Fig. 3A-D). In addition, IF staining results proved that the cleavage of the classic apoptotic protein caspase 3 in neurons was reduced in the ISRIB-treated group compared with the SCI group at $7 \mathrm{dpi}$ (Fig. 3E, F), indicating that ISRIB can limit the apoptotic cascade after SCI.

\section{SCI-Induced Protein Translocation and Inhibition of Protein Synthesis Are Reversed by ISRIB Treatment}

Subsequently, nuclear and cytoplasmic proteins were isolated from spinal cord tissues, and it was found that the translocation of ATF4 from the cytosol to the nucleus was enhanced after SCI but that ISRIB administration significantly inhibited this translocation (Fig. 4A, B). Detection of newly synthesized puromycin-labeled peptides also revealed that protein translation, another process involving eIF $2 \alpha$ phosphorylation, was impaired after SCI, but this impairment was alleviated by ISRIB treatment after SCI (Fig. 5E, F). The assembly of stress 


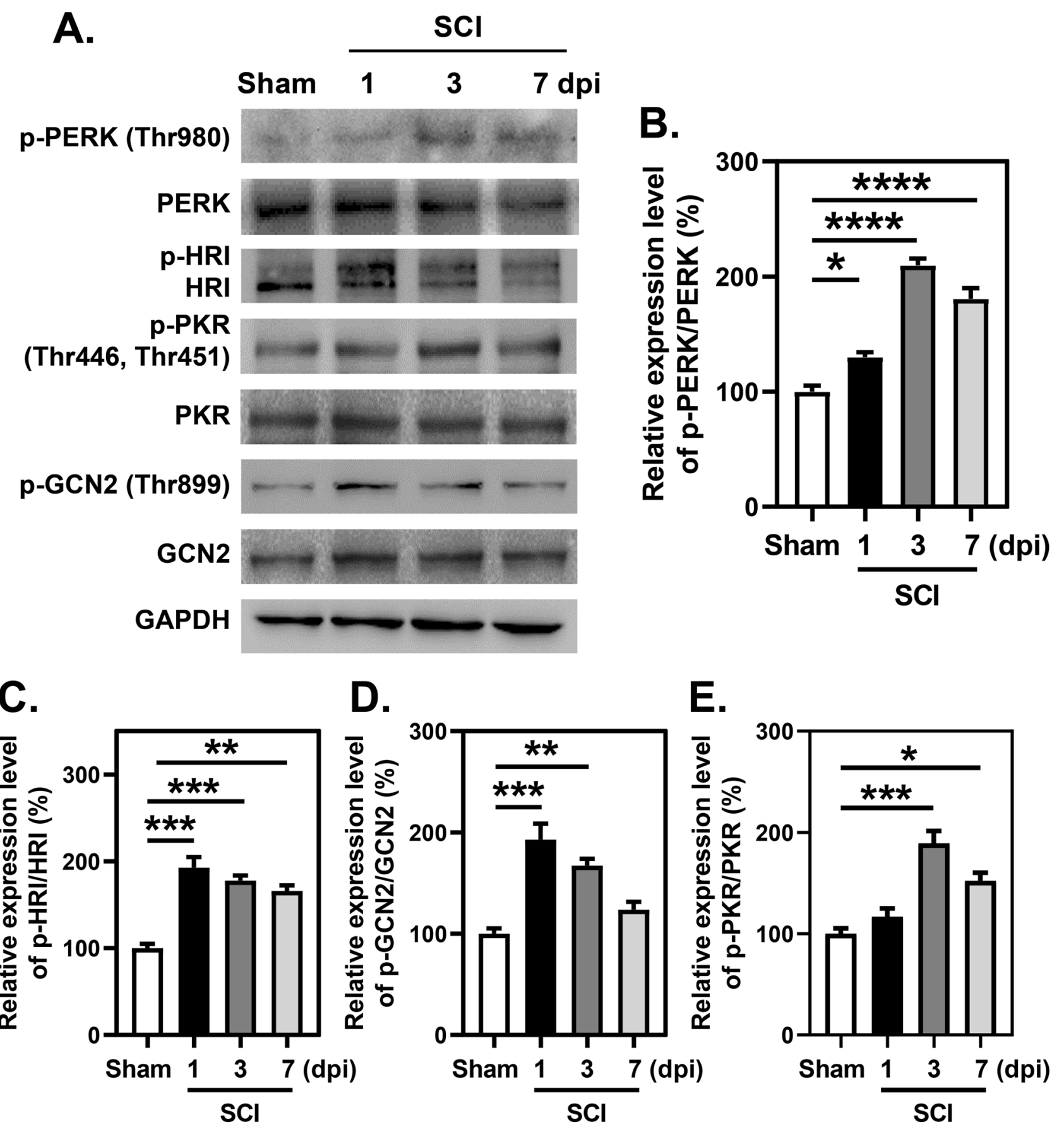

Fig. 1 Activation of ISR in spinal cord after SCI. A-E WB analysis and quantitative analysis of phosphorylation of PERK, HRI, GCN2, and PKR at indicated time points following SCI. $n=3$ (each group contains 3 mice). B-E One-way ANOVA and Tukey's multiple com-

granules (SGs) in the cytoplasm was evaluated by IF staining for TIA-1 and analysis of the cytosolic translocation of TIA-1, FUS, and TDP-43 after SCI by WB. It was found that ISRIB treatment significantly decreased the cytosolic localization of SG assembly associated proteins in the spinal cord, especially in neurons, after SCI (Figs. 4C, D and 5A-D). parisons test. $* P<0.05$, sham vs. 1 dpi, sham vs. $7 \mathrm{dpi} ; * * P<0.01$, sham vs. $3 \mathrm{dpi}$, sham vs. $7 \mathrm{dpi}$; $* * * P<0.001$, sham vs. $1 \mathrm{dpi}$, sham vs. $3 \mathrm{dpi} ; * * * * P<0.0001$, sham vs. $3 \mathrm{dpi}$, sham vs. $7 \mathrm{dpi}$

\section{ISRIB Treatment Ameliorates Pathological Deterioration and Improves Locomotor Function After SCI}

H\&E staining, footprint analysis, and the BMS scoring system were then used to evaluate the therapeutic effects 


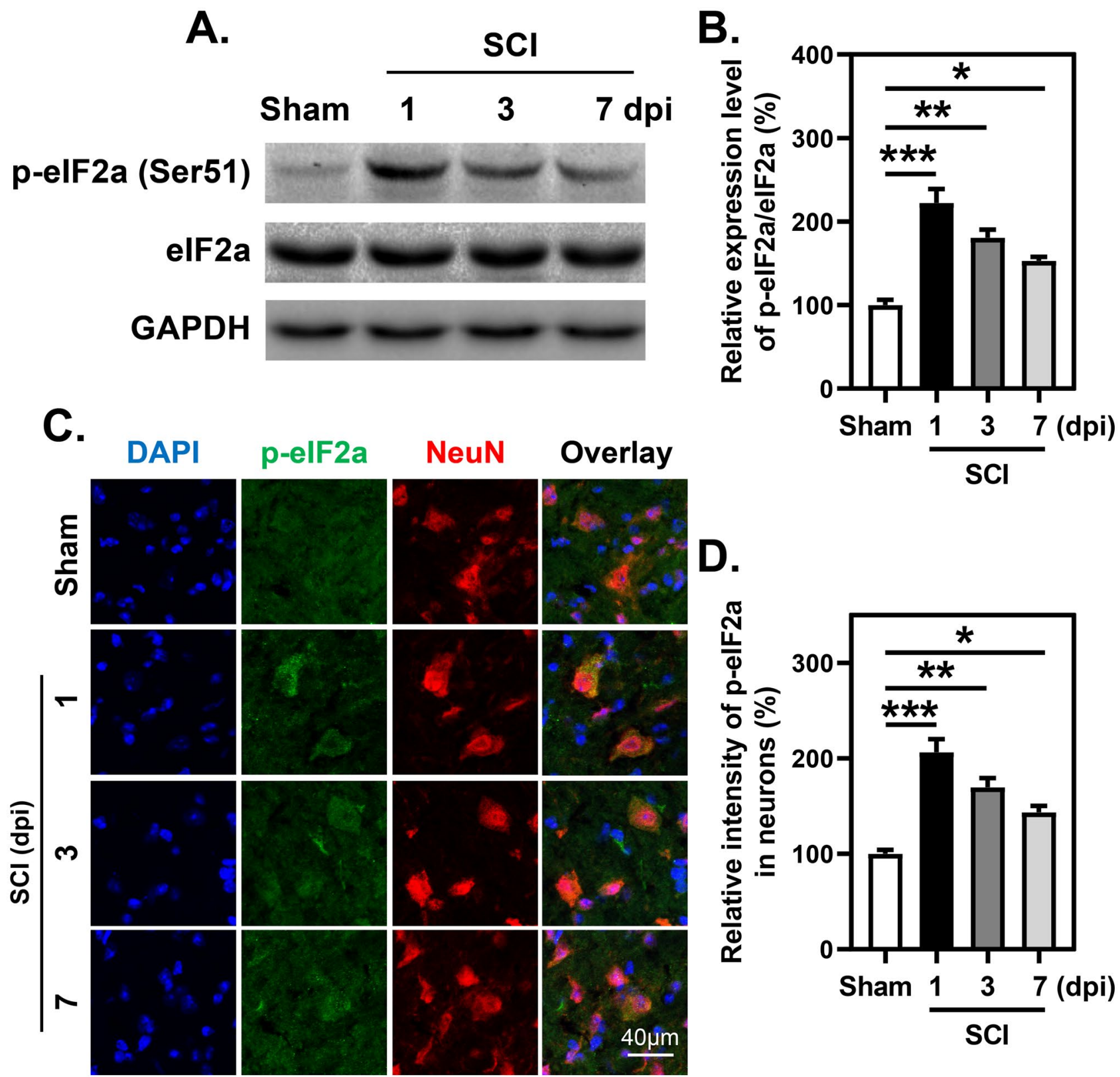

Fig. 2 Phosphorylation of eIF2 $\alpha$ in spinal cord and neurons after SCI. $\mathbf{A}$ and $\mathbf{B}$ WB analysis and quantitative analysis of phosphorylation of eIF2 $\alpha . n=3$. C and D Representative images and quantitative analysis of spinal cord transection stained with anti-p-eIF $2 \alpha$ and anti-NeuN

of ISRIB treatment after SCI. H\&E staining revealed that ISRIB treatment reduced the loss of neurons in the anterior horn and the formation of tissue cavities around the epicenter of the spinal cord at $28 \mathrm{dpi}$ (Fig. 6A). Footprint analysis showed that ISRIB treatment improved the locomotor function of the hind limbs at $28 \mathrm{dpi}$, as indicated by an improved trajectory (Fig. 6B). In addition, the recovery of behavioral function was promoted by ISRIB administration after SCI, and the BMS scores of ISRIB-treated mice were antibodies at indicated time points following SCI. $n=3$. B and D One-way ANOVA and Tukey's multiple comparisons test. $* P<0.05$, sham vs. $7 \mathrm{dpi} ; * * P<0.01$, sham vs. $3 \mathrm{dpi} ; * * * P<0.001$, sham vs. $1 \mathrm{dpi}$

significantly higher than those of untreated mice at $28 \mathrm{dpi}$ (Fig. 6C, D).

\section{Discussion}

The continuous expansion of secondary injury after SCI, as an incurable disease, greatly affects later functional recovery. Current therapeutic strategies for SCI mainly involve the 


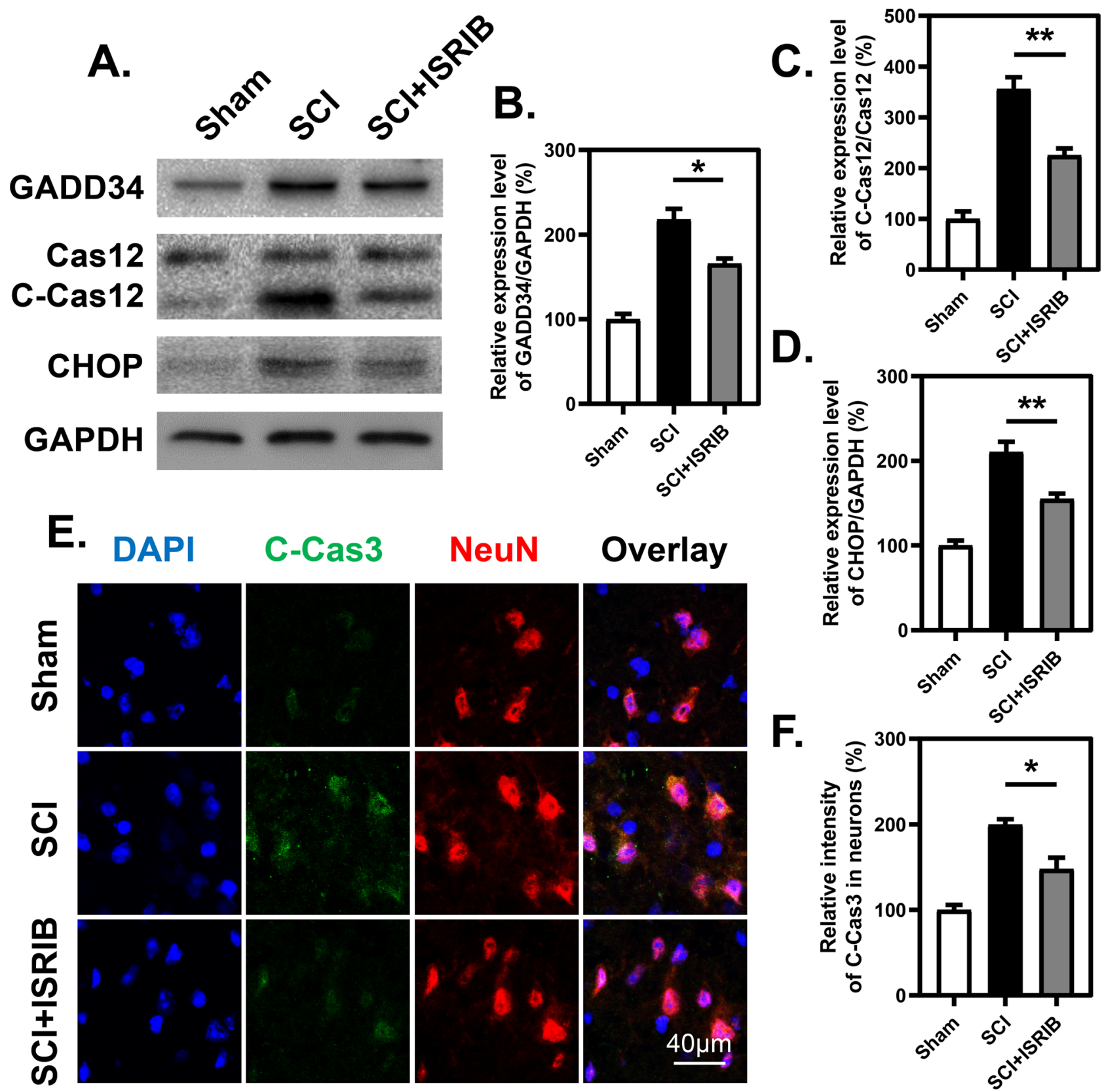

Fig. 3 ISRIB inhibits ISR signal and apoptosis after SCI. A-D WB analysis and quantitative analysis of ISR-related proteins (GADD34 and $\mathrm{CHOP}$ ) and proteotoxicity-associated apoptotic protein cleavedcaspase 12 (C-Cas12) at $7 \mathrm{dpi}$ with or without treatment of ISRIB. $n=3$. $\mathbf{E}$ and $\mathbf{F}$ Representative images and quantitative analysis of spi-

following: (1) attenuation of neuronal loss; (2) promotion of neuronal network reconstruction; and (3) stem cell transplantation (Guo et al. 2012; Das et al. 2013; Lai et al. 2013; Sabelstrom et al. 2013). Studies have highlighted that the number of neurons in the injured segment of the spinal cord determines functional recovery after SCI. Thus, we aimed to inhibit apoptotic cascades in neurons and improve neuronal function after SCI. nal cord transection stained with anti-cleaved-caspase 3 (C-Cas3) and anti-NeuN antibodies at $7 \mathrm{dpi}$ with or without treatment of ISRIB. $n=3$. B-D and F One-way ANOVA and Tukey's multiple comparisons test. $* P<0.05, * * P<0.01, \mathrm{SCI}$ vs. SCI + ISRIB

Previous studies have verified that perturbation of ER homeostasis, which triggers rapid ERSR and is involved in morphological deterioration, occurs in different SCI models (Valenzuela et al. 2012; Ohri et al. 2013; Liu et al. 2015). In addition, overactivation of oxidative stress caused by excessive generation of reactive oxygen species and ischemia/ hypoxia caused by blood vessel loss have been demonstrated to be involved in secondary degeneration following SCI and 


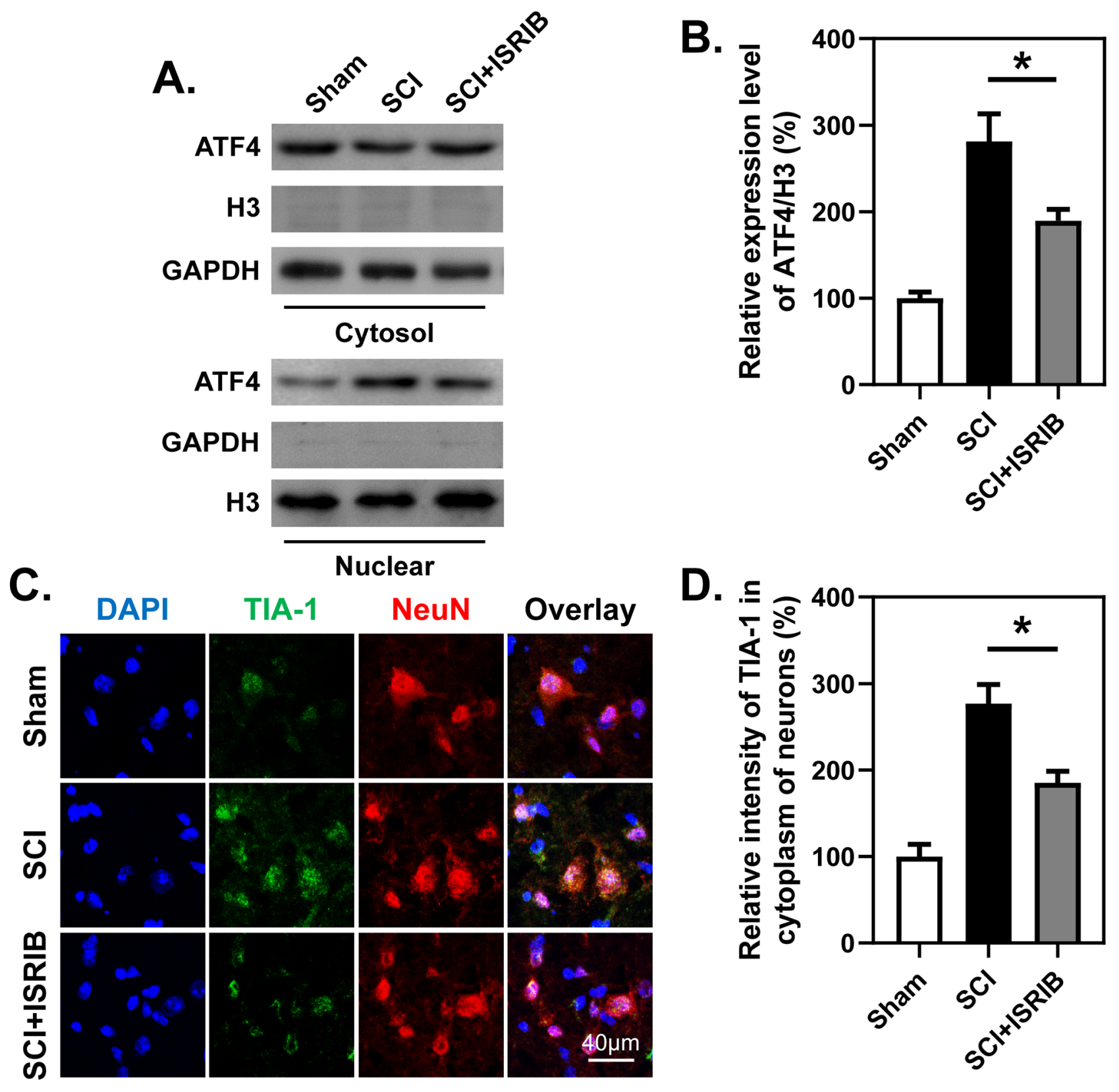

Fig. 4 ISRIB decreases nuclear translocation of ATF4 and formation of stress granules after SCI. A and B WB analysis and quantitative analysis of nuclear translocation of ATF4 at 7 dpi with or without treatment of ISRIB. $n=3$. C and D Representative images and quan-

may be therapeutic targets ( $\mathrm{Li}$ et al. 2017; Wang et al. 2020a, b). Nevertheless, the role of the ISR, which responds to multiple stresses after SCI, remains to be clarified. Here, we found that the four branches of the ISR (PERK, HRI, GCN2, PKR) were all activated after SCI, as indicated by increased autophosphorylation of each protein. In addition, the activation peaks of the four branches exhibited incomplete overlap in our study, indicating the complexity of the microenvironment after SCI. titative analysis of spinal cord transection stained with anti-TIA-1 and anti-NeuN antibodies at 7 dpi with or without treatment of ISRIB. $n=3$. B and D One-way ANOVA and Tukey's multiple comparisons test. $* P<0.05$, SCI vs. SCI + ISRIB

Subsequently, it was determined that the ISR downstream molecule eIF2 $\alpha$ was activated after SCI, and the results showed that the phosphorylation of eIF $2 \alpha$ at Ser51 was enhanced after SCI. Strikingly, eIF2 $\alpha$ phosphorylation peaked at $1 \mathrm{dpi}$, and then eIF2 $\alpha$ activation was maintained a low level, suggesting that the ISR was strongly stimulated in the acute phase and then continued to be activated in the subacute phase after SCI. It is widely accepted that transient activation of the ISR can lead to reprogramming of protein 


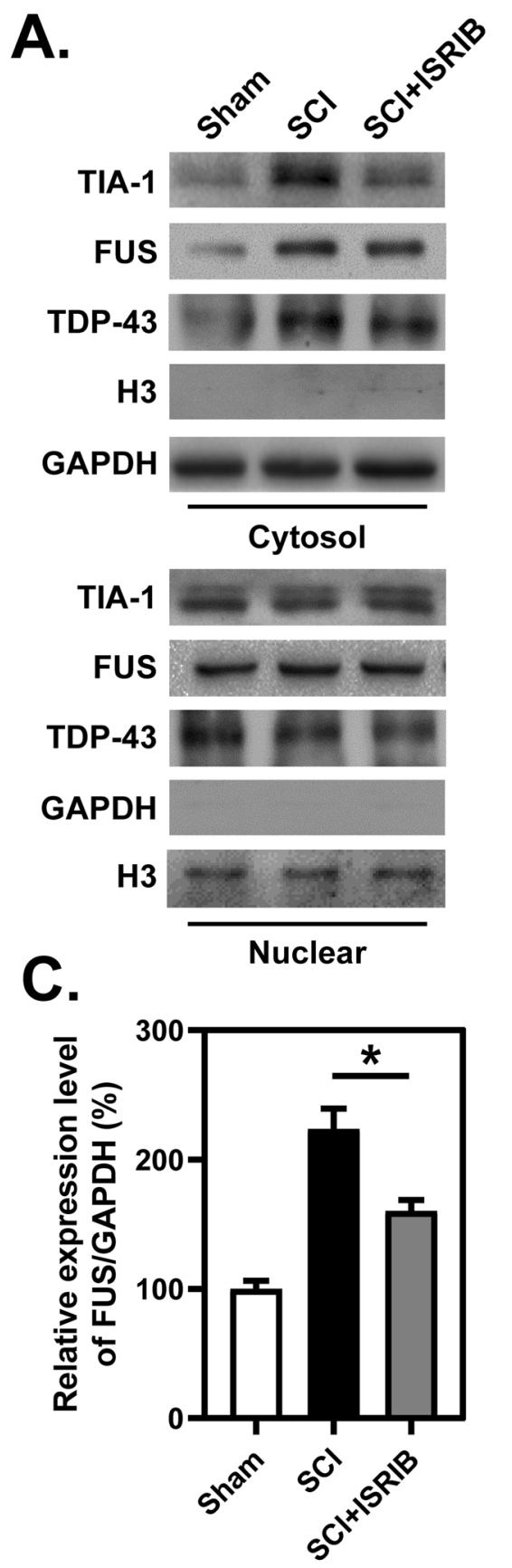

Fig. 5 ISRIB dwindles cytosolic location of RNA-binding proteins and enhances protein synthesis after SCI. A-D WB analysis and quantitative analysis of nuclear translocation of TIA-1, FUS, and TDP-43 at 7 dpi with or without treatment of ISRIB. $n=3$. $\mathbf{E}$ and $\mathbf{F}$

transcription and regulate gene expression in response to stress and resist stress, while persistence and abnormal activation of the ISR can affect neural function and even trigger cellular apoptosis by limiting the translation of proteins required for neural function (Fan et al. 2013; Lopez-Erauskin et al. 2018; Kapur et al. 2020). Therefore, strategies that can maintain ISR-induced stress resistance in the acute phase
$\mathbf{F}$.
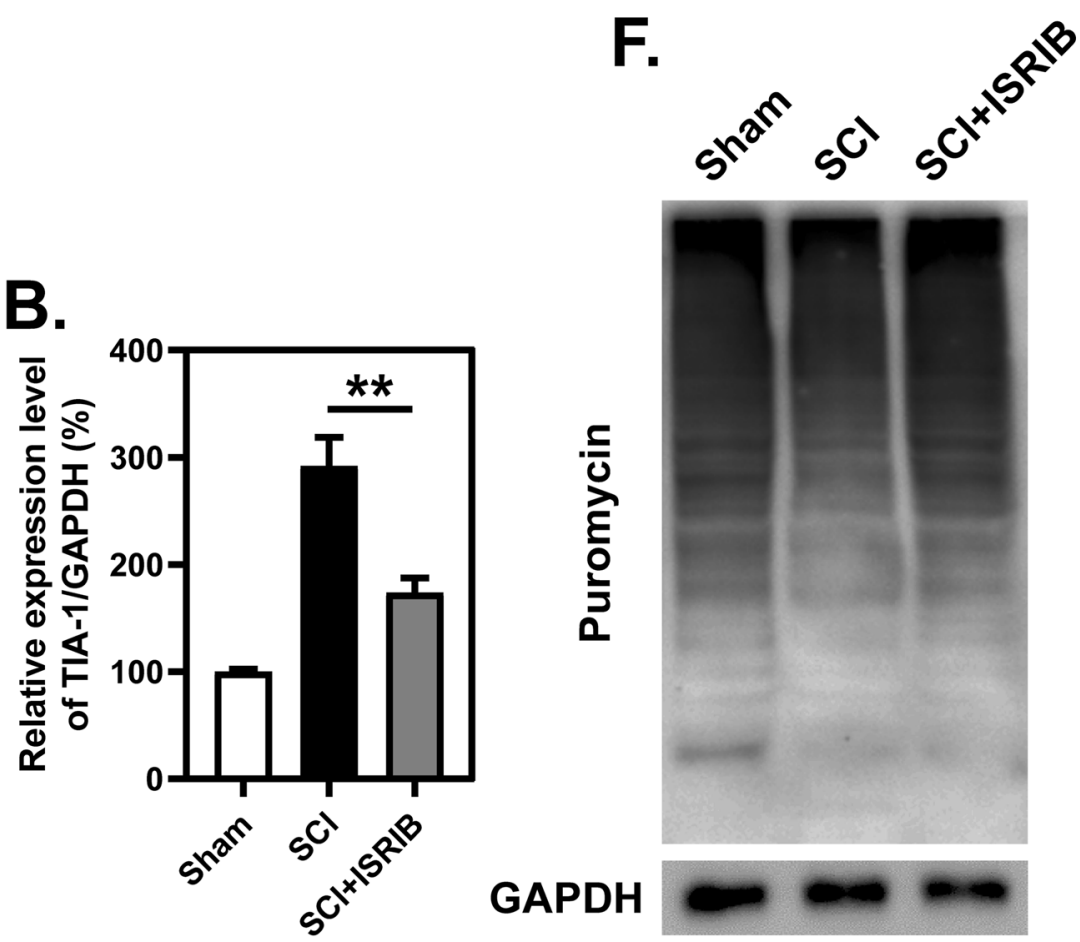

GAPDH

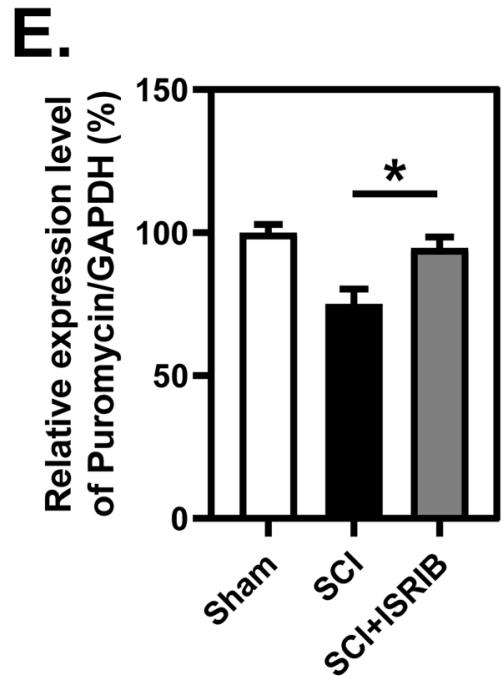

WB analysis and quantitative analysis of puromycin-labeled newly synthesis peptides at $7 \mathrm{dpi}$ with or without treatment of ISRIB. $n=3$. B-E One-way ANOVA and Tukey's multiple comparisons test. ${ }^{*} P<$ $0.05, * * P<0.01$, SCI vs. SCI + ISRIB

and prevent ISR-induced neurodegeneration in the subacute phase after SCI are promising.

Thus, the ISR inhibitor ISRIB is a therapeutic candidate due to its unique target. ISRIB serves as an activator of eIF2B, and the assembly of eIF2B can be promoted under moderate stress to reactivate eIF2 and thus restore global translation (Costa-Mattioli and Walter 2020). Recent studies 

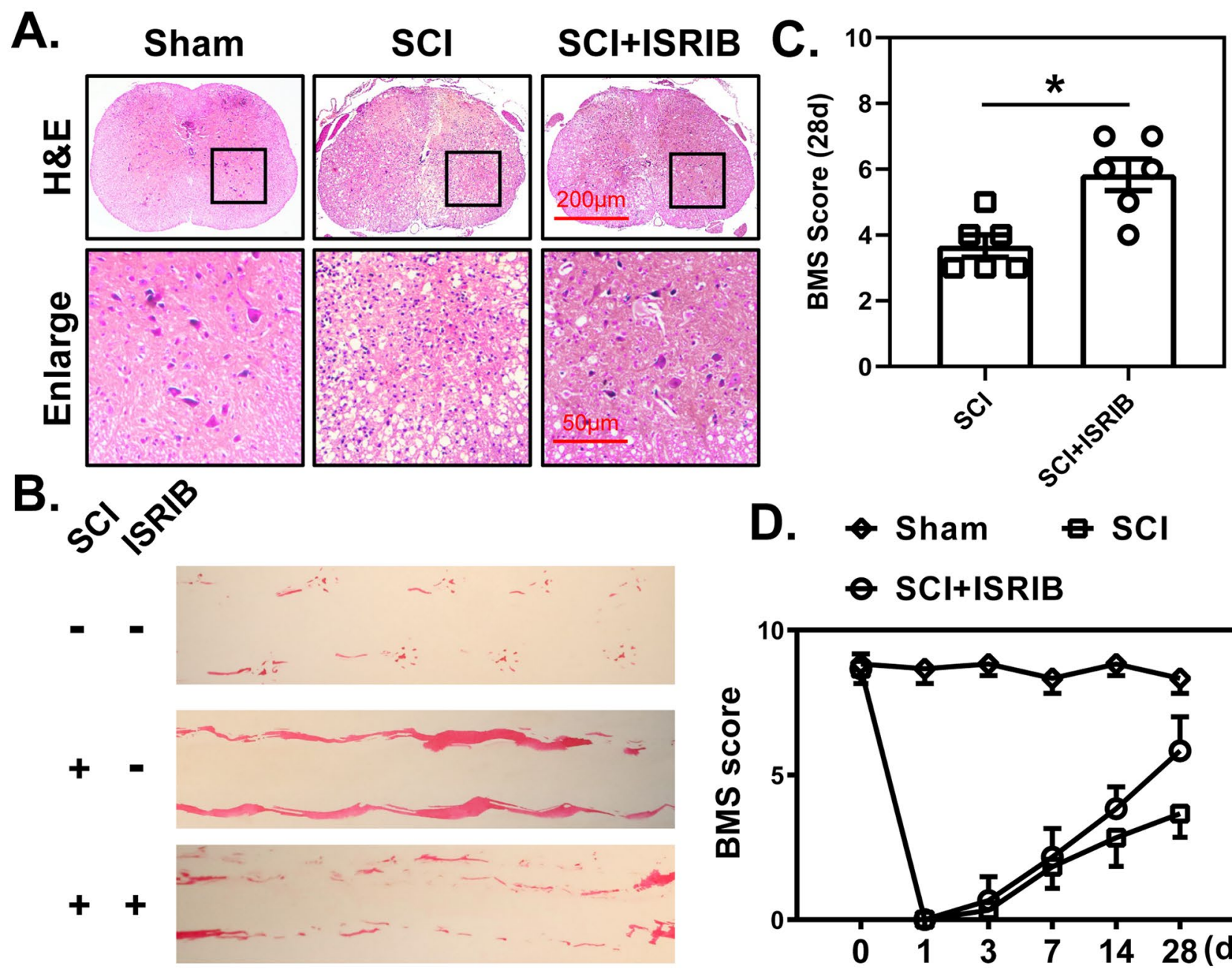

Fig. 6 ISRIB improves histopathology and locomotor function after SCI. A Representative images of spinal cord transection stained with H\&E at 28 dpi. $n=6$. B Representative images of locomotion tra-

have highlighted the significance of ISRIB in enhancing cognition and ameliorating cognitive deficits after brain injury (Tsai et al. 2018), but its role in other types of nerve injuries and neurodegenerative diseases needs to be further determined. After administration of ISRIB to mice subjected to SCI, eIF $2 \alpha$ downstream signaling was significantly suppressed, as characterized by decreased translocation of ATF4 to the nucleus and subsequent downregulation of GADD34 and CHOP expression. By labeling nascent peptide chains with puromycin, we found that protein translation was decreased after SCI and that protein translation was promoted after ISRIB administration, confirming that the decrease in protein translation after SCI was attributed to redistribution of translation caused by eIF $2 \alpha$ phosphorylation and nuclear translocation of its downstream molecule ATF4.

As a result of multiple stresses and obstacles to protein translation, SGs can be assembled as a result of the jectories of mice hind limbs. $n=6$. $\mathbf{C}$ and $\mathbf{D}$ Quantitative analysis of BMS score from 0-28 dpi and at $28 \mathrm{dpi}$ among different groups. $n=6$. Mann-Whitney test. $* P<0.05$, SCI vs. SCI + ISRIB

translocation of RNAs and RNA-binding proteins to the cytoplasm to arrest mRNA and then inhibit translation (Nahm et al. 2020). It has been reported that the formation of SGs is induced in the brain after traumatic brain injury (TBI), another type of CNS injury, and that SGs might aggregate. Genetic and pharmacological induction of SG clearance alleviates neural degeneration after TBI (Anderson et al. 2018). Our results demonstrated for the first time that SG assembly related RNA-binding proteins were also abundant in the cytoplasm in the spinal cord, especially in neurons, in the subacute phase after SCI, suggesting delayed clearance of SGs after SCI. Nevertheless, ISRIB treatment reduced the levels of RNA-binding proteins in the cytoplasm and even the expression levels of apoptosis-associated genes at $7 \mathrm{dpi}$, indicating that ISRIB contributes to inhibiting the SCI-triggered cytotoxic response involved in the pathogenesis of neurodegenerative diseases (Wang et al. 2020a, b). 


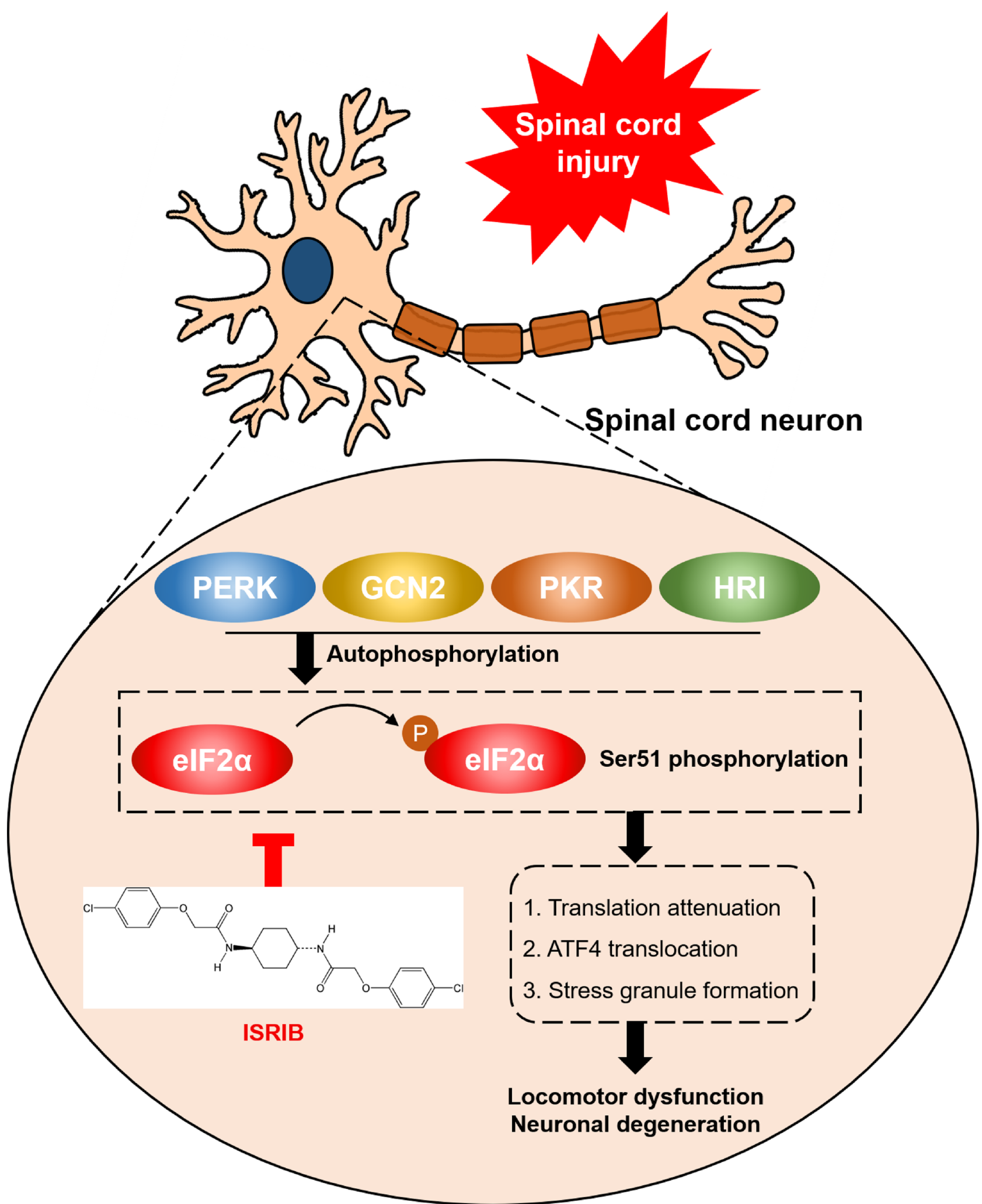

Fig. 7 Following SCI, ISR signal is activated by autophosphorylation of PERK, GCN2, PKR, and HRI, which phosphorylates Ser51 in eIF2 $\alpha$ to attenuate global translation and promote nuclear translo- cation of ATF4 and stress granule formation. Nevertheless, treatment of ISRIB after SCI alleviates ISR-induced effects to delay neuronal degeneration and improve locomotor function 
Finally, we observed pathologic and functional improvement in ISRIB-treated mice subjected to SCI through H\&E staining, BMS scoring, and footprint analysis. However, our research has the following limitations: (1) We solely assessed variations in neuronal fate after SCI. There are other neural cells in the CNS, and whether ISRIB directly targets neurons to exert a therapeutic effect requires further clarification. (2) The therapeutic window of ISRIB after SCI needs to be further confirmed to achieve better therapeutic results. (3) Whether ISRIB treatment has potential therapeutic significance for types of SCI other than contusion SCI, such as spinal cord hemisection and transection, remains defined. Collectively, our results confirm that the ISR is activated after SCI and prove that the cytosolic localization of RNA-binding proteins in the spinal cord, especially in neurons, is increased after SCI. Our work highlights the role of the ISR in the subacute phase after SCI and supports the use of ISRIB as a promising therapeutic agent after SCI (Fig. 7).

Acknowledgements All authors listed are grateful for the technical and instrument support provided by the large-scale instrument management platform of Hunan Normal University.

Author Contribution LC and XJS were responsible for experimental design; LC wrote the manuscript; XJS edited the manuscript; LC, XYL, JC, and HZL were responsible for animal experiments; LC, GPW, and GHW collected the data and made analyses; LC and XYL confirmed and purchased reagents and antibodies; LC, XYL, and XJS organized and arranged the figures.

Funding This work was supported by research grants from the Scientific Research Project of Hunan Provincial Department of Education (18C0029) and Scientific Research Project of Hunan Provincial Health and Family Planning Commission (20201751, 20201755).

Availability of Data and Materials The data that support the findings of this study are available from the corresponding author upon reasonable request.

\section{Declarations}

Ethics Approval and Consent to Participate All experimental procedures were approved by Medical Ethics Committee of Hunan People's Hospital (First Affiliated Hospital of Hunan Normal University) and were performed in accordance with the National Institutes of Health guide for the care and use of Laboratory animals.

Consent for Publication All authors listed agree to publish the manuscript.

Competing Interests The authors declare no competing interests.

Open Access This article is licensed under a Creative Commons Attribution 4.0 International License, which permits use, sharing, adaptation, distribution and reproduction in any medium or format, as long as you give appropriate credit to the original author(s) and the source, provide a link to the Creative Commons licence, and indicate if changes were made. The images or other third party material in this article are included in the article's Creative Commons licence, unless indicated otherwise in a credit line to the material. If material is not included in the article's Creative Commons licence and your intended use is not permitted by statutory regulation or exceeds the permitted use, you will need to obtain permission directly from the copyright holder. To view a copy of this licence, visit http://creativecommons.org/licenses/by/4.0/.

\section{References}

Anderson EN, Gochenaur L, Singh A et al (2018) Traumatic injury induces stress granule formation and enhances motor dysfunctions in ALS/FTD models. Hum Mol Genet 27:1366-1381. https://doi. org/10.1093/hmg/ddy047

Arai S, Varkaris A, Nouri M et al (2020) MARCH5 mediates NOXAdependent MCL1 degradation driven by kinase inhibitors and integrated stress response activation. Elife 9. https://doi.org/10. 7554/eLife.54954

Costa-Mattioli M, Walter P (2020) The integrated stress response: from mechanism to disease. Science 368. https://doi.org/10.1126/science. aat5314

Das A, Gt Wallace, Reiter RJ et al (2013) Overexpression of melatonin membrane receptors increases calcium-binding proteins and protects VSC4.1 motoneurons from glutamate toxicity through multiple mechanisms. J Pineal Res 54:58-68. https://doi.org/10. 1111/j.1600-079X.2012.01022.x

Fan J, Long H, Li Y et al (2013) Edaravone protects against glutamateinduced PERK/EIF2alpha/ATF4 integrated stress response and activation of caspase-12. Brain Res 1519:1-8. https://doi.org/10. 1016/j.brainres.2013.04.037

Griffin DE (2003) Immune responses to RNA-virus infections of the CNS. Nat Rev Immunol 3:493-502. https://doi.org/10.1038/nri1105

Guo X, Ayala JE, Gonzalez M et al (2012) Tissue engineering the monosynaptic circuit of the stretch reflex arc with co-culture of embryonic motoneurons and proprioceptive sensory neurons. Biomaterials 33:5723-5731. https://doi.org/10.1016/j.biomaterials. 2012.04 .042

Kapur M, Ganguly A, Nagy G et al (2020) Expression of the neuronal tRNA n-Tr20 regulates synaptic transmission and seizure susceptibility. Neuron 108(193-208):e199. https://doi.org/10. 1016/j.neuron.2020.07.023

Kong H, Reczek CR, McElroy GS et al (2020) Metabolic determinants of cellular fitness dependent on mitochondrial reactive oxygen species. Sci Adv 6. https://doi.org/10.1126/sciadv.abb7272

Kroemer G, Marino G, Levine B (2010) Autophagy and the integrated stress response. Mol Cell 40:280-293. https://doi.org/10.1016/j. molcel.2010.09.023

Krukowski K, Nolan A, Frias ES et al (2020) Small molecule cognitive enhancer reverses age-related memory decline in mice. Elife 9. https://doi.org/10.7554/eLife.62048

Lai BQ, Wang JM, Duan JJ et al (2013) The integration of NSC-derived and host neural networks after rat spinal cord transection. Biomaterials 34:2888-2901. https://doi.org/10.1016/j.biomaterials. 2012.12.046

Li H, Zhang X, Qi X et al (2019) Icariin inhibits endoplasmic reticulum stress-induced neuronal apoptosis after spinal cord injury through modulating the PI3K/AKT signaling pathway. Int J Biol Sci 15:277-286. https://doi.org/10.7150/ijbs.30348

Li Y, Lucas-Osma AM, Black S et al (2017) Pericytes impair capillary blood flow and motor function after chronic spinal cord injury. Nat Med 23:733-741. https://doi.org/10.1038/nm.4331

Liu S, Sarkar C, Dinizo M et al (2015) Disrupted autophagy after spinal cord injury is associated with ER stress and neuronal cell death. Cell Death Dis 6:e1582. https://doi.org/10.1038/cddis.2014.527 
Liu S, Li Y, Choi HMC et al (2018) Lysosomal damage after spinal cord injury causes accumulation of RIPK1 and RIPK 3 proteins and potentiation of necroptosis. Cell Death Dis 9:476. https://doi. org/10.1038/s41419-018-0469-1

Liu Y, Ye Y (2011) Proteostasis regulation at the endoplasmic reticulum: a new perturbation site for targeted cancer therapy. Cell Res 21:867-883. https://doi.org/10.1038/cr.2011.75

Lopez-Erauskin J, Tadokoro T, Baughn MW et al (2018) ALS/FTDlinked mutation in FUS suppresses intra-axonal protein synthesis and drives disease without nuclear loss-of-function of FUS. Neuron 100(816-830):e817. https://doi.org/10.1016/j.neuron.2018.09.044

Malvezzi AM, Arico M, Souza-Melo N et al (2020) GCN2-like kinase modulates stress granule formation during nutritional stress in Trypanosoma cruzi. Front Cell Infect Microbiol 10:149. https:// doi.org/10.3389/fcimb.2020.00149

Miller KD, Schnell MJ, Rall GF (2016) Keeping it in check: chronic viral infection and antiviral immunity in the brain. Nat Rev Neurosci 17:766-776. https://doi.org/10.1038/nrn.2016.140

Nahm M, Lim SM, Kim YE et al (2020) ANXA11 mutations in ALS cause dysregulation of calcium homeostasis and stress granule dynamics. Sci Transl Med 12. https://doi.org/10.1126/scitranslmed.aax3993

Ohri SS, Hetman M, Whittemore SR (2013) Restoring endoplasmic reticulum homeostasis improves functional recovery after spinal cord injury. Neurobiol Dis 58:29-37. https://doi.org/10.1016/j. nbd.2013.04.021

Park HW, Park H, Ro SH et al (2014) Hepatoprotective role of Sestrin2 against chronic ER stress. Nat Commun 5:4233. https://doi.org/ $10.1038 /$ ncomms 5233

Rabouw HH, Langereis MA, Anand AA et al (2019) Small molecule ISRIB suppresses the integrated stress response within a defined window of activation. Proc Natl Acad Sci U S A 116:2097-2102. https://doi.org/10.1073/pnas.1815767116

Rubiano AM, Carney N, Chesnut R et al (2015) Global neurotrauma research challenges and opportunities. Nature 527:S193-197. https://doi.org/10.1038/nature16035

Sabelstrom H, Stenudd M, Reu P et al (2013) Resident neural stem cells restrict tissue damage and neuronal loss after spinal cord injury in mice. Science 342:637-640. https://doi.org/10.1126/ science. 1242576

Surget A, Tanti A, Leonardo ED et al (2011) Antidepressants recruit new neurons to improve stress response regulation. Mol Psychiatry 16:1177-1188. https://doi.org/10.1038/mp.2011.48

Tsai JC, Miller-Vedam LE, Anand AA et al (2018) Structure of the nucleotide exchange factor eIF2B reveals mechanism of memoryenhancing molecule. Science 359. https://doi.org/10.1126/science. aaq0939
Tsarouchas TM, Wehner D, Cavone L et al (2018) Dynamic control of proinflammatory cytokines Il-1beta and Tnf-alpha by macrophages in zebrafish spinal cord regeneration. Nat Commun 9:4670. https://doi.org/10.1038/s41467-018-07036-w

Valenzuela V, Collyer E, Armentano D et al (2012) Activation of the unfolded protein response enhances motor recovery after spinal cord injury. Cell Death Dis 3:e272. https://doi.org/10.1038/cddis. 2012.8

Wagner FB, Mignardot JB, Le Goff-Mignardot CG et al (2018) Targeted neurotechnology restores walking in humans with spinal cord injury. Nature 563:65-71. https://doi.org/10.1038/ s41586-018-0649-2

Wang F, Li J, Fan S et al (2020a) Targeting stress granules: a novel therapeutic strategy for human diseases. Pharmacol Res 161:105143. https://doi.org/10.1016/j.phrs.2020.105143

Wang H, Zheng Z, Han W et al (2020b) Metformin promotes axon regeneration after spinal cord injury through inhibiting oxidative stress and stabilizing microtubule. Oxid Med Cell Longev 2020:9741369. https://doi.org/10.1155/2020/9741369

Wang S, Wu J, Zeng YZ et al (2017) Necrostatin-1 mitigates endoplasmic reticulum stress after spinal cord injury. Neurochem Res 42:3548-3558. https://doi.org/10.1007/s11064-017-2402-x

Wang XJ, Peng CH, Zhang S et al (2019) Polysialic-acid-based micelles promote neural regeneration in spinal cord injury therapy. Nano Lett 19:829-838. https://doi.org/10.1021/acs.nanolett.8b04020

Wang Y, Wu W, Wu X et al (2018) Remodeling of lumbar motor circuitry remote to a thoracic spinal cord injury promotes locomotor recovery. Elife 7. https://doi.org/10.7554/eLife.39016

Zhou R, Ma Y, Tao Z et al (2020) Melatonin inhibits glucose-induced apoptosis in osteoblastic cell line through PERK-eIF2alpha-ATF4 pathway. Front Pharmacol 11:602307. https://doi.org/10.3389/ fphar.2020.602307

Zhu PJ, Khatiwada S, Cui Y et al (2019) Activation of the ISR mediates the behavioral and neurophysiological abnormalities in Down syndrome. Science 366:843-849. https://doi.org/10.1126/science. aaw5185

Zyryanova AF, Weis F, Faille A et al (2018) Binding of ISRIB reveals a regulatory site in the nucleotide exchange factor eIF2B. Science 359:1533-1536. https://doi.org/10.1126/science.aar5129

Publisher's Note Springer Nature remains neutral with regard to jurisdictional claims in published maps and institutional affiliations. 